\title{
Theorie des Sperrschicht-Photoeffekts II
}

\author{
Von Kurt Lehovec \\ (Z. Naturforschg. 2 a, 398-403 [1947]; eingegangen am 27. April 1946)
}

\begin{abstract}
Eine Beziehung zwischen Photospannung, Photostrom, Beleuchtungsstärke, Lichtfrequenz, Temperatur, Sperrschicht- und Halbleiter-Eigenschaften wird abgeleitet. Daraus ergibt sich unter anderem: 1. Die Elektrode an der Sperrschicht wird bei Belichtung bei Überschußhalbleitern positiv, bei Defekthalbleitern negativ. - 2. Der Kurzschlußstrom ist der Beleuchtungsstärke proportional. - 3. Die Leerlaufspannung ist zunächst der Beleuchtungsstärke, später dem log derselben annähernd proportional. Der Ubergang erfolgt bei Leerlaufspannungen von der Größenordnung der Temperaturspannung $\left(25 \mathrm{mV}\right.$ bei $\left.20^{\circ} \mathrm{C}\right)$. - 4. Die Photokennlinie steht in engem Zusammenhang mit der Dunkelkennlinie. - 5. Die spektrale Verteilung der Vorderwandzellen ist von der Absorption im Halbleiter, der Dicke der Sperrschicht, der Randfeldstärke und der Temperatur abhängig.
\end{abstract}

W haben kürzlich die Grundlagen einer Theorie des Sperrschicht-Photoeffekts entwickelt und durch Differentialgleichungen formuliert ${ }^{1}$. In vorliegender Arbeit werden wir die Differentialgleichungen integrieren und die Lösung diskutieren. Besonders günstig für die Integration ist der Umstand, daß für die erstrebte Beziehung zwischen Photospannung, Photostrom und Beleuchtungsstärke nur der Potentialverlauf in der Sperrschicht in der unmittelbaren Nachbarschaft der Metallelektrode maßgebend ist. Die Rechnung ist für Defekthalbleiter durchgeführt; bei Überschußhalbleitern kann sinngemäß verfahren werden. Die wichtigsten Aussagen über SperrschichtPhotozellen mit Überschußhalbleitung sind am Schluß der Arbeit angeführt. Die experimentellen Ergebnisse an Sperrschicht-Photozellen werden in einer weiteren Arbeit vom Standpunkt der vorliegenden Theorie diskutiert werden.

1. Ableitung einer Beziehung zwischen Photospannung, Photostrom und Lichtintensität

Für das Auftreten des Sperrschicht-Photoeffekts sind

a) Störstellenhalbleitung (und zwar entweder praktisch ausschließliche Überschußhalbleitung oder praktisch ausschließliche Defekthalbleitung) und

b) Auslösung von je einem Elektron und Defektelektron durch ein absorbiertes Lichtquant

${ }^{1}$ K. L e hove c, Z. Naturforschg. 1, 258 [1946]. wesentlich. An der Grenze zu einem Metall wird dem Halbleiter eine bestimmte Elektronen- und Defektelektronen-Konzentration aufgeprägt ${ }^{1}$.

Im folgenden betrachten wir ausschließliche Defekthalbleiter. Technisch interessante Verhältnisse entstehen, wenn die Dichte der Defektelektronen am Rande um so viele Größenordnungen geringer ist als im Innern des Halbleiters, daß der Widerstand der gesamten Anordnung praktisch durch den Widerstand der „Sperrschicht", d.h. der Randschicht mit wesentlicher Verarmung der Defektelektronen, bestimmt ist. Die Sperrschichtdicke definieren wir durch

$$
n=f \cdot N^{-} \text {für } x=d .
$$

(x Ortskoordinate, vom Metallrand aus gezählt, $d$ Sperrschichtdicke, $n$ Dichte der Defektelektronen, $N^{-}$Dichte der dissoziierten Störstellen, $f$ Zahlenfaktor, etwa zwischen 0,1 und $0,5^{2}$.)

Dem Konzentrationsgefälle der Defektelektronen in der Sperrschicht wird durch ein elektrisches Potential das Gleichgewicht gehalten. Wird das Gleichgewicht gestört (z. B. bei Stromdurchgang oder bei Belichtung), so tritt

$$
E=V_{R}-\mathfrak{B} \ln \frac{n_{d}}{n_{R}}
$$

als resultierender Spannungsabfall nach außen in Erscheinung. ( $V_{R}$ elektrischer Spannungsabfall

${ }^{2}$ Der Zahlenwert ist praktisch bedeutungslos, weil sein Einfluß auf $d$ nur einige Prozente beträgt und der Spannungsabfall am halbleiterseitigen Teil der Sperrschicht gegenüber demjenigen am metallseitigen Teil vollständig zurücktritt. 
an der Sperrschicht, $\mathfrak{B}=k T / e ; k$ BoltzmannKonstante, $T$ absolute Temperatur, $e$ Elementarladung, $n_{d}, n_{R}$, Dichte der Defektelektronen am halbleiterseitigen bzw. metallseitigen Rand der Sperrschicht.) Das elektrische Potential in der Sperrschicht wird durch den Überschuß der dissoziierten Störstellen über die Defektelektronen bewirkt:

$$
\frac{d^{2} V}{d x^{2}}=-\frac{4 \pi e}{\varepsilon}\left(n-N^{-}\right) .
$$

( $V$ elektrisches Potential in der Sperrschicht, $\varepsilon$ Dielektrizitätskonstante des Halbleiters). Den Nullpunkt des elektrischen Potentials legen wir an den halbleiterseitigen Rand der Sperrschicht. Der Höchstwert des elektrischen Potentials liegt am metallseitigen Rand der Sperrschicht ${ }^{3}$.

Im stationären Zustand fließen sämtliche bei Belichtung in der Sperrschicht ausgelösten Defektelektronen ab, da infolge der geringen Defektelektronen-Dichten keine nennenswerte Rekombination erfolgen kann. Daher ist für $x \leqq d$

$$
Z^{h v}-\frac{1}{e} \frac{d i}{d x}=0 .
$$

Es bedeuten

$$
Z^{h v}=\frac{J}{h v} \frac{1}{x_{0}} \exp \left(-\frac{x}{x_{0}}\right)=\frac{Z}{x_{0}} \exp \left(-\frac{x}{x_{0}}\right)
$$

( $J$ Beleuchtungsstärke, $h v$ Energie eines Lichtquants, $Z$ Gesamtzahl der einfallenden Lichtquanten je $\mathrm{cm}^{2}$ und sec, $x_{0}$ Absorptionslänge des Halbleiters) die Zahl der pro sec und $\mathrm{cm}^{3}$ absorbierten

${ }^{3}$ Die Raumladung in der Sperrschicht besteht praktisch aus den Störstellen. Sind diese infolge der extremen Verarmung an Defektelektronen vollständig dissoziiert („Erschöpfungsrandschicht“), so ergibt sich statt (3):

$$
\frac{d^{2} V}{d x^{2}} \approx \frac{4 \pi e}{\varepsilon} N
$$

( $N$ Dichte der Störstellen) und integriert:

$$
\begin{gathered}
\frac{d V}{d x} \approx-\frac{4 \pi e}{\varepsilon} \int_{x}^{d} N d x \\
V \approx \frac{4 \pi e}{\varepsilon}\left[\int_{x}^{d} N x d x-x \int_{x}^{d} N d x\right] .
\end{gathered}
$$

(Die Feldstärke am halbleiterseitigen Rand der Sperrschicht kann gegenüber derjenigen am metallseitigen Rand vernachlässigt werden!)
Lichtquanten, und

$$
i=-n e b \cdot d V / d x-e D \cdot d n / d x
$$

( $b$ Beweglichkeit, $D=\mathfrak{B} b$ Diffusionskonstante der Defektelektronen) die Stromdichte der Defektelektronen ${ }^{4}$.

Die Integration von (4) ergibt mit (5):

$$
Z \exp \left(-x / x_{0}\right)+i / e=Z \exp \left(-d / x_{0}\right)+I / e .
$$

Die Integrationskonstante wurde durch den Defektelektronen-Strom am halbleiterseitigen Rand der Sperrschicht $I$ ausgedrückt. (7) stellt unter Berücksichtigung von (6) eine lineare inhomogene Differentialgleichung erster Ordnung für $n$ dar. Das allgemeine Integral ist

$$
n=[c+F] \exp \left(-V_{/} \mathfrak{B}\right)
$$

mit der Abkürzung

$$
\begin{array}{r}
F=\frac{Z}{D} \int_{0}^{x}\left\{\exp \left(-t / x_{0}\right)-\exp \left(-d / x_{0}\right)-I / Z e\right\} \\
\cdot \exp (+V / \mathfrak{B}) d t
\end{array}
$$

Die Dichte der Defektelektronen am metallseitigen Rand der Sperrschicht ist durch das Gleichgewicht mit dem Metall festgelegt ${ }^{5}$. Aus dieser Randbedingung ergibt sich die Integrationskonstante von (8):

$$
c=n_{R} \exp \left(V_{R} / \mathfrak{V}\right) .
$$

Für die Dichte der Defektelektronen am halbleiterseitigen Rand der Sperrschicht ergibt sich aus (8) und (10)

$$
n_{d}=n_{R} \exp \left(V_{R} / \mathfrak{B}\right)+F_{x=d} .
$$

Die wesentlichen Beiträge zu $F$ werden von den Stellen, wo $V$ am größten ist, also von den Werten um $x \approx 0$, geliefert. Daher können wir im Integrand von (9) mit guter Näherung

$$
V \approx V_{R}-\mathfrak{F}_{R} \cdot x
$$

${ }^{4}$ Auch bei einem praktisch ausschließlichen Defekthalbleiter bildet sich bei Belichtung der Sperrschicht ein Elektronenstrom aus, der den Defektelektronenstrom zum ortsunabhängigen Gesamtstrom ergänzt.

5 Die Konzentration der Defektelektronen am metall seitigen Rand der Sperrschicht kann auch von der Feldstärke (z. B. Feldemission oder Erniedrigung der Austrittsarbeit durch polarisierbare Dipolschichten) abhängen, ohne die folgenden Überlegungen zu stören. 
setzen. $\mathfrak{F}_{R}(>0)$ stellt die elektrische Feldstärke am metallseitigen Rand der Sperrschicht dar. Da die obere Integrationsgrenze infolge des exponentiellen Abfalls des Integranden belanglos ist, ergibt sich aus (9) und (12)

$$
F_{x=d} \approx \frac{Z}{D} \exp \left(\frac{V_{R}}{\mathscr{D}}\right)
$$

$\cdot\left\{\left(\frac{1}{x_{0}}+\frac{\mathfrak{F}_{R}}{\mathfrak{B}}\right)^{-1}-\left[\exp \left(-\frac{d}{x_{0}}\right)+\frac{I}{Z e}\right]\left(\frac{\mathfrak{F}_{R}}{\mathfrak{B}}\right)^{-1}\right\}$.

Nach einigen Umrechnungen ergibt (11) unter Benützung von (2) und (13) die folgende Beziehung zwischen der Spannung an der Sperrschicht, dem Defektelektronenstrom am halbleiterseitigen Rand der Sperrschicht und der Zahl der einfallenden Lichtquanten

$$
\begin{gathered}
Z e\left[\left(1+\mathfrak{B} / \mathfrak{F}_{R} x_{0}\right)^{-1}-\exp \left(-d / x_{0}\right)\right]-I \\
=n_{R} e b \mathfrak{F}_{R}[\exp (-E / \mathfrak{B})-1]
\end{gathered}
$$

bzw. nach Einführung der Beleuchtungsstärke

$$
\begin{gathered}
J /(h \nu) \cdot e\left[\left(1+\mathfrak{V} / \mathfrak{E}_{R} x_{0}\right)^{-1}-\exp \left(-d / x_{0}\right)\right]-I \\
=n_{R} e b \mathfrak{E}_{R}[\exp (-E / \mathfrak{B})-1] .
\end{gathered}
$$

Solange der Spannungsabfall an der Sperrschicht groß gegen den Spannungsabfall an der übrigen Halbleiterschicht ist, können wir $\boldsymbol{E}$ mit der Klemmenspannung der Sperrschicht-Photozelle identifizieren. Bei fehlender Beleuchtung und bei Beleuchtung mit stark absorbiertem Licht $\left(x_{0} \ll d\right)$ hat der Defektelektronen-Strom bereits an der Stelle $x=d$ den Wert des ortsunabhängigen Gesamtstroms erreicht. Bei Beleuchtung mit schwach absorbiertem Licht $\left(x_{0}>d\right)$ werden die außerhalb der Sperrschicht durch Lichtabsorption gebildeten Defektelektronen und Elektronen vorwiegend rekombinieren, so daß wir auch in diesem Fall I mit dem ortsunabhängigen Gesamtstrom identifizieren können ${ }^{6}$.

\footnotetext{
${ }^{6}$ Die Verteilung der Elektronen in der Sperrschicht brauchen wir nicht $\mathrm{zu}$ berücksichtigen, solange ihre Dichte $-\boldsymbol{n}$ innerhalb der Sperrschicht weit unter $n_{\boldsymbol{d}}$ bleibt. (Der Index deutet auf Elektronen hin.) Dies ist, wie ohne Beweis mitgeteilt sei, für Beleuchtungsstärken $J \ll n_{d}{ }^{-} D \cdot h v / d$ erfüllt. Bei diesen Beleuchtungsstärken kann der Raumladungsbeitrag der Elektronen in (3) fortgelassen werden, und es ist kein nennenswerter Beitrag des Elektronenstroms zum Gesamtstrom am halbleiterseitigen Rand der Sperrschicht zu erwarten.
}

\section{Diskussion}

Mit (15) ist ein Zusammenhang zwischen der Klemmenspannung $E$, dem Strom $I$ und der Belichtungsstärke $J$ gew onnen. Außerdem treten die Randfeldstärke $\mathfrak{夭}_{R}$, die Sperrschichtdicke $d$ und Größen, die als anderweitig bekannt angesehen werden können, auf. $E$, $\mathfrak{夭}_{R}$ und $d$ sind nicht unabhängig voneinander, sondern infolge (2) und (3) (vergl. $3 \mathrm{~b}, 3 \mathrm{c}$ ) verknüpft. Daher können $\mathfrak{F}_{R}$ und $d$ (bei vorgegebener Verteilung der Störstellen in der Sperrschicht) als (bekannte) Funktionen von $E$ aufgefaßt werden.

Von besonderem Interesse sind die folgenden Spezialfälle von (15):

\section{A. Sperrschicht-Gleichrichter}

Kennlinie der unbelichteten Sperrschicht $(J=0)$ : Dieser Sonderfall entspricht der Theorie des Sperrschicht-Gleichrichters, die in erweitertem Umfang bereits von W. Schottky und E. $\mathrm{S} \mathrm{penke} \mathrm{F}^{7}$ behandelt wurde. Wir können uns daher auf einige kurze Angaben beschränken.

Für $E \approx 0$ folgt aus (15) der sogenannte ,Nullwiderstand“" zu :

$$
R_{0}=\frac{\mathfrak{B}}{n_{R} e b \mathfrak{S}_{R}} .
$$

Für $E \ll-\mathfrak{B}$ ergibt sich

$$
I \approx-n_{R} e b \mathfrak{F}_{R} \exp (-E / \mathfrak{B}) .
$$

$I$ ist negativ (entsprechend einem Defektelektronen-Strom vom Halbleiter zum Metall) und nimmt im wesentlichen exponentiell mit der Klemmenspannung zu („Flußrichtung“) ${ }^{8}$.

Für $E \gg \mathfrak{B}$ ergibt sich

$$
I \approx n_{R} e b \mathfrak{F}_{R} .
$$

$I$ ist positiv und nimmt verhältnismäßig langsam mit der Spannung zu (,,Sperrichtung“) ${ }^{\text {9. }}$

7 Wiss. Veröff. Siemens-Werke 18, 225 [1939].

8 Dies gilt natürlich nur so lange, als der Widerstand der Sperrschicht groß gegen den Widerstand der neutralen Halbleiterschicht ist, was für $E \simeq-\mathfrak{B} \ln \left(n_{a} / n_{R}\right)$ nicht mehr erfüllt ist!

9 In diesem Bereich bestehen zwischen Theorie und Experiment größere Unterschiede, die von W. S chottky (Z. Physik 118, 534 [1942]) auf die „Paßleitfähigkeit", welche mit der statistischen Verteilung der Störstellen in der Sperrschicht zusammenhängt, zurückgeführt wurden. 


\section{B. Sperrschicht-Photozelle}

a) Kurzschluß $(E=0)$ : Nach (14) ist der Kurzschlußstrom (Strom bei kurzgeschlossenen Elektroden) der Beleuchtungsstärke proportional und entspricht einem Strom in „Sperrichtung“. Für die Quantenausbeute des Kurzschlußstroms ergibt sich:

$$
\delta=I_{k} / Z e=\left(1+\mathfrak{B} / \mathfrak{F}_{R} x_{0}\right)^{-1}-\exp \left(-d / x_{0}\right)
$$

Mit $E$ ist auch $\mathfrak{F}_{R}, d$ und somit die spektrale Verteilung des Kurzschlußstroms von der Be-

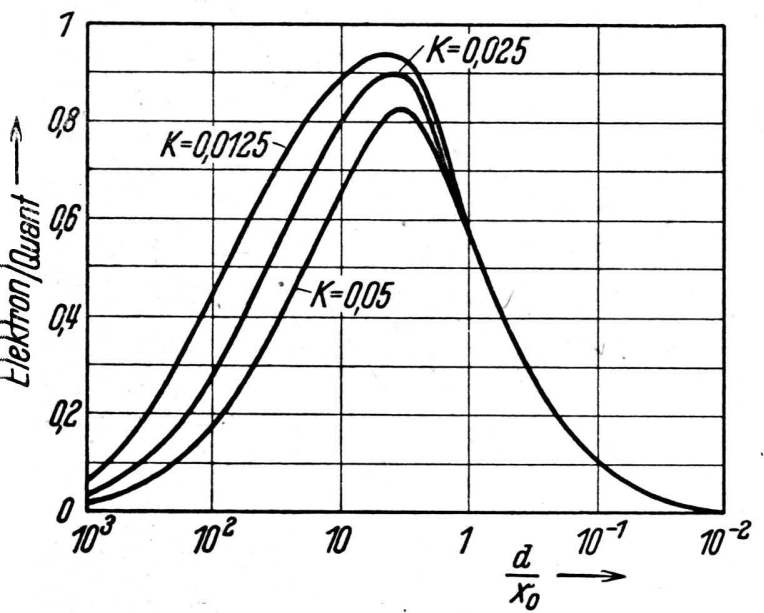

Abb. 1. Zur spektralen Verteilung der Quantenausbeute der Vorderwand-Sperrschichtzelle

( $k=0,0025$ entspricht $T=20^{\circ} \mathrm{C}, d \xi=1$ Volt).

Abszisse: Verhältnis der Sperrschichtdicke zur Absorptionslänge des Halbleiters. Ordinate: Quantenausbeute in $E l_{\text {. }}$ Quant.

leuchtungsstärke unabhängig ${ }^{10}$. In Abb. 1 haben wir die Quantenausbeute als Funktion von $d / x_{0}$ aufgetragen; als einziger Parameter bleibt $\mathfrak{B} / \mathfrak{F}_{R} d$.

Das Maximum der Ausbeute liegt bei Absorptionslängen, die einige Zehntel der Sperrschichtdicke betragen: Fast alle durch das Licht ausgelösten Defektelektronen werden durch das elektrische Feld in den Halbleiter geführt. Der Abfall der Ausbeute gegen große Absorptionslängen $\left(x_{0}>d\right.$; lange Wellen) beruht auf der geringen Absorption des Lichts innerhalb der Sperrschicht. Der Abfall gegen kleine Absorptionslängen $\left(x_{0} \ll d\right.$; kurze Wellen) kommt dadurch zu-

10 Vorausgesetzt, daß durch das Licht in der Sperrschicht nicht zusätzlich Störstellen ionisiert werden und sich die Dielektrizitätskonstante nicht ändert. stande, daß sich ein zu starkes Konzentrationsgefälle in der Sperrschicht ausbildet, wodurch ein Teil der Deféktelektronen zur Elektrode getrieben wird. Da die Sperrschichtdicke in der Größenordnung $10^{-5} \mathrm{~cm}$ liegt, sind hohe Ausbeuten nur an der Absorptionskante des Halbleiters zu erwarten.

b) Leerlauf $(I=0)$ : Nach (14) beträgt die Leerlaufspannung (Spannung, die an den Elektroden der belichteten Photozelle auftritt, wenn kein Strom entnommen wird) :

$$
\begin{aligned}
& E_{L}=-\mathfrak{B} \ln \left\{1+\frac{Z}{n_{R} b \mathfrak{F}_{R}}\right. \\
& \left.\cdot\left[\left(1+\mathfrak{B} / \mathfrak{F}_{R} x_{0}\right)^{-1}-\exp \left(-d / x_{0}\right)\right]\right\} .
\end{aligned}
$$

Das Vorzeichen entspricht einer Spannung in „Flußrichtung“. Die Leerlaufspannung ist nach (20) zunächst der Beleuchtungsstärke, später dem log derselben annähernd proportional. Das Übergangsgebiet liegt bei Spannungen von der Größenordnung der Temperaturspannung $\mathfrak{B}$. Da sich Sperrschichtdicke und Randfeldstärke mit der Spannung ändern, ist die spektrale Verteilung der Leerlaufspannung von der Beleuchtungsstärke abhängig.

c) Photokennlinie ( $J=$ const): Der Zusammenhang zwischen Photospannung und Photostrom bei konstanter Belichtung kann entweder unmittelbar aus (15) entnommen oder über die Dunkelkennlinie ermittelt werden.

Aus (15) ist $\mathrm{zu}$ ersehen, daß verschiedene Wertepaare $J, I$ zur gleichen Klemmenspannung führen, wenn für sie der Ausdruck

$$
\frac{J e}{h \nu} \cdot A-I
$$

der gleiche ist. Hierbei wurde

$$
A=\left(1+\frac{\mathfrak{B}}{\mathfrak{E}_{R} \cdot x_{0}}\right)^{-1}-\exp \left(-\frac{d}{x_{0}}\right)
$$

gesetzt. Insbesondere entspricht dem Wertepaar $J, I$ das Wertepaar

$$
J^{*}=0, I^{*}=I-\frac{J e}{h \nu} A
$$

Die Photospannung bei einer bestimmten Beleuchtungsstärke $J$ und einem bestimmten Photostrom $I$ ist daher gleich der Spannung, die sich aus der Flußkennlinie der unbelichteten Sperrschicht für den Strom $I^{*}$ entnehmen läßt. 


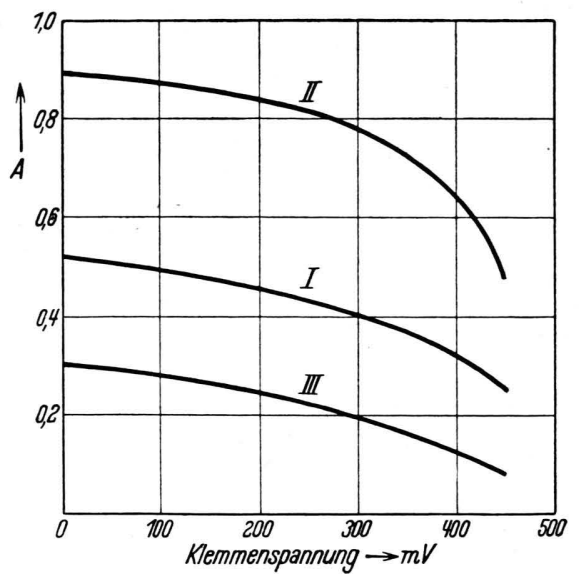

Abb. 2. Abhängigkeit des Faktors $A$ von der Klemmenspannung. Konstante Störstellendichte $N=4 \cdot 10^{15} \mathrm{~cm}^{-3}$ (vollständige Dissoziation); $n_{R}=10^{7} \mathrm{~cm}^{-3}, \varepsilon=10$.

\begin{tabular}{cc} 
Kurve & Absorptionslä \\
\hline I & $10^{-6} \mathrm{~cm}$ \\
II & $10^{-5} \mathrm{~cm}$ \\
III & $10^{-4} \mathrm{~cm}$
\end{tabular}

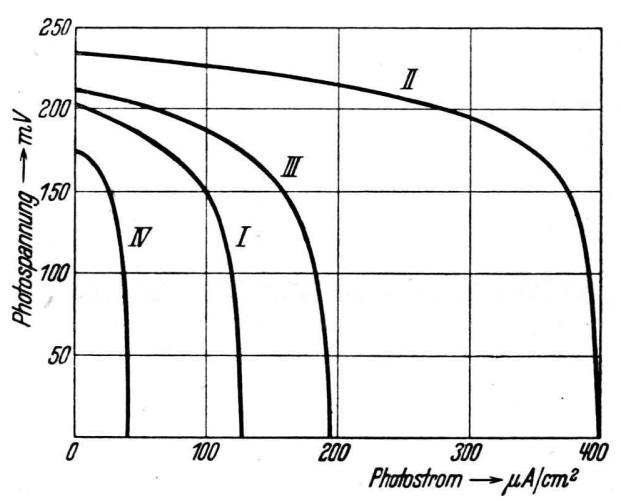

Abb. 3. Photokennlinien. Konstante Störstellendichte $N=4 \cdot 10^{15} \mathrm{~cm}^{-3}$ (vollständige Dissoziation); $n_{R}=10^{7} \mathrm{~cm}^{-3} ; \varepsilon=10 ; b=1 \mathrm{~cm}^{2} /$ Volt.sec.

Kurve $\begin{gathered}\text { Beleuchtungs- } \\ \text { stärke }\end{gathered}$ Wellenlänge Absorptionslänge

$\begin{array}{rlll}\text { I } & 10^{-3} \mathrm{~W} \text { Watt } / \mathrm{cm}^{2} & 3,0 \cdot 10^{-5} \mathrm{~cm} & 10^{-6} \mathrm{~cm} \\ \text { II } & 10^{-3} \mathrm{Watt} / \mathrm{cm}^{2} & 5,5 \cdot 10^{-5} \mathrm{~cm} & 10^{-5} \mathrm{~cm} \\ \text { III } & 10^{-3} \mathrm{Watt} / \mathrm{cm}^{2} & 8,0 \cdot 10^{-5} \mathrm{~cm} & 10^{-4} \mathrm{~cm} \\ \text { IV } & 10^{-4} \mathrm{Watt} / \mathrm{cm}^{2} & 5,5 \cdot 10^{-5} \mathrm{~cm} & 10^{-5} \mathrm{~cm}\end{array}$

In Abb. 2 ist die Abhängigkeit des Faktors $A$ von der Klemmenspannung unter Annahme ortsunabhängiger Störstellen-Dichte in der Sperrschicht ${ }^{11}$ für drei Wellenlängen und Absorptions-

${ }_{11}$ Bei konstanter Dichte und vollständiger Dissoziation der Störstellen ist (vergl. Fußnote ${ }^{3}$ ) :

$$
d=\sqrt{\frac{\varepsilon V_{R}}{2 \pi e \mathrm{~N}}} \text { und } \S_{R}=\sqrt{\frac{8 \pi e N V_{R}}{\varepsilon}}=\frac{2 V_{R}}{d} \text {. }
$$

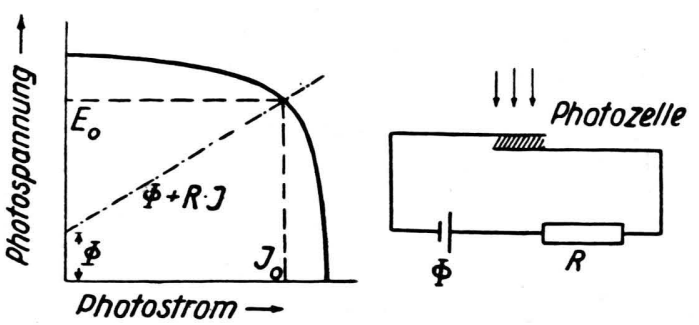

Abb. 4. Bestimmung von Photospannung und Photostrom aus der Photokennlinie bei nebenstehender Schaltung.

längen eingezeichnet. (Die Zahlenwerte der Abb.2 u. 3 entsprechen etwa den Verhältnissen bei SelenSperrschichtzellen.) Abb. 3 zeigt die Photokennlinien, die sich mit Hilfe der in Abb. 2 eingetragenen $A$-Werte aus (15) ergeben. Die Kurven I, II, III beziehen sich auf gleiche Beleuchtungsstärke, jedoch verschiedene Wellenlängen. Die Kurven II und IV unterscheiden sich nur in der zugrunde gelegten Beleuchtungsstärke. Die Kurzschlußströme verhalten sich entsprechend den Beleuchtungsstärken wie $10: 1$, wogegen sich die Leerlaufspannungen infolge der annähernd logarithmischen Abhängigkeit von der Beleuchtungsstärke nur relativ wenig unterscheiden.

Wird die Photozelle mit einem Außenwiderstand $R$ in einen Stromkreis geschaltet, so kann die Photospannung und der Photostrom bei vorgegebener Belichtung aus (15) und der Gleichung

$$
-E=R I
$$

ermittelt werden. Stets ist

$$
E \leqq 0 \quad \text { und } \quad 0 \leqq I<\frac{J}{h \nu} e ;
$$

die Photospannung entspricht stets einer Spannung in Flußrichtung und der Photostrom einem Strom in Sperrichtung. Ein graphisches Verfah- ren zur Auflösung von (15) und (23) (wobei noch zusätzlich eine EMK im Außenkreis angenommen wurde) ist aus Abb. 4 ersichtlich.

Zum Abschluß einige Bemerkungen über den Sperrschicht-Photoeffekt bei Überschußhalbleitern. An Stelle von (15) ergibt sich:

$$
\begin{array}{r}
\frac{J e}{h \nu}\left[\left(1-\frac{\mathfrak{B}}{\mathfrak{E}_{R} \cdot x_{0}}\right)^{-1}-\exp \left(-\frac{d}{x_{0}}\right)\right]+I \\
=-{ }^{-} n_{R} e^{-} b \mathfrak{F}_{R}\left[\exp \left(\frac{E}{\mathfrak{B}}\right)-1\right] .
\end{array}
$$


$-n_{R}$ und $-b$ sind Randkonzentration und Beweglichkeit der Elektronen. Die Randfeldstärke $\mathfrak{r}_{R}$ ist negativ, da die Raumladung in der Sperrschicht durch positiv geladene Störstellen bedingt ist. $I$ und $E$ haben gegenüber (15) die Vorzeichen geändert. Daher sind Flußrichtung und Sperrichtung bei Überschußhalbleitern gegenüber Defekthalbleitern vertauscht; bei Überschußhalbleitern ist die belichtete Elektrode positiv, der Photostrom fließt vom Halbleiter zur Elektrode. Der Zusammenhang zwischen Flußkennlinie und Photokennlinie und die übrigen Beziehungen des zweiten Abschnitts können ohne weiteres übernommen werden.

Bezeichnet man mit $\mathfrak{F}_{R}, I$ und $E$ die Absolut-Beträge von Randfeldstärke, Photostrom und Photo- spannung, mit $n$ bzw. $b$ die Dichte bzw. Beweglichkeit der für die Leitung im Halbleiter-Innern wesentlichen Ladungsträger, so gilt für Defektund Überschußhalbleiter:

$$
\begin{array}{r}
\frac{J}{h \nu} e\left[\left(1+\frac{\mathfrak{B}}{\mathfrak{F}_{R} x_{0}}\right)^{-1}-\exp \left(-\frac{d}{x_{0}}\right)\right]-I \\
=n_{R} e b \mathfrak{S}_{R}\left[\exp \left(\frac{E}{\mathfrak{V}}\right)-1\right] .
\end{array}
$$

Unsere Ausführungen bezogen sich auf die gebräuchlichen Vorderwandzellen, wie aus der Annahme (5) über die Verteilung der Lichtintensität in der Sperrschicht hervorgeht. Bei Hinterwandzellen ändert sich lediglich der Ausbeutefaktor $A$, für welchen vor allem die Filterwirkung der vorgelagerten Halbleiterschicht maßgebend wird.

\title{
Über einen photoelektrischen Rausch-Effekt
}

\author{
Von Walter Reichelt und August Becker \\ Aus dem Physikalischen Institut der Universität Heidelberg \\ (Z. Naturforschg. 2 a, 403-409 [1947] eingegangen am 26. Juli 1946 *)
}

\begin{abstract}
Es ist bekannt, daß in Gleichstromkreisen, die einen Halbleiter enthalten, Stromschwankungen, sog. Rausch-Effekte, auftreten können, welche der beliebigen Verstärkung eines durch äußere Einwirkungen auf den Halbleiter hervorgerufenen Stromeffekts eine unliebsame Grenze setzen und daher im allgemeinen als Störungsquelle betrachtet werden. Die vorliegende Untersuchung betrifft die demgegenüber neue Erfahrung, daß geeignete lichtelektrisch empfindliche Halbleiter bzw. Isolatoren, die kein nachweisbares Dunkelrauschen zeigen und die wir Photoleiter nennen wollen, bei Belichtung im Gleichstromkreis niederfrequente Schwankungen zeigen, die der normalen Wechselstromverstärkung zugänglich sind und dann, im Lautsprecher als Rauschen abgehört oder anderweitig gemessen, ein hochempfindliches Nachweismittel für schwächste, insbesondere auch ultrarote Lichtwirkungen darstellen.
\end{abstract}

\section{Erste Beobachtungen}

$\mathrm{B}^{\mathrm{i}}$ ei Versuchen über die lichtelektrische Leitfähigkeit gewisser $\mathrm{CdS}-\mathrm{Cu}-\mathrm{Phosphore} \mathrm{im}$ Gleichstromkreis zeigte es sich, daß der bei konstanter Belichtung derselben auftretende Photostrom sich nicht auf konstante Werte einstellte, wie dies etwa bei normal präparierten $\mathrm{ZnS}$ - oder CdS-Phosphoren der Fall ist, sondern Schwankungen um einen Mittelwert aufwies, deren Amplitude mit wachsender Bestrahlungsintensität sehr beträchtliche Werte annahm. Diese Schwankungen blieben auch nach sorgfältigster Ausschaltung aller apparativen Störungsmöglichkeiten unver-

* Bei der Heidelberger Akademie der Wissenschaften deponiert am 16. November 1945. ändert erhalten, so daß die Ursache im Phosphor selbst zu suchen war.

Die Meßweise ist die, daß man einen den Photoleiter enthaltenden Kondensator mit einer Batterie (etwa $100 \mathrm{~V}$ ) und einem großen Widerstand $\left(10^{6}\right.$ bis $10^{7} \Omega$ ) in Reihe schaltet und direkt oder indirekt den bei Belichtung des Photoleiters am Widerstand auftretenden Spannungsabfall, etwa durch den von einem empfindlichen Galvanometer angezeigten Photostrom, kenntlich macht. Es ergibt sich dabei das schematisch in Abb. 1 veranschaulichte Bild. Der Dunkelstrom spielt keine Rolle, da seine Größe bei unseren in Betracht kommenden Präparaten hinter einem Tausendstel des Photostroms zurückbleibt. Letzterer steigt prak- 